\title{
345.
}

\section{ON THE INFLEXIONS OF THE CUBICAL DIVERGENT PARABOLAS.}

[From the Quarterly Journal of Pure and Applied Mathematics, vol, vi. (1864), pp. 199-203.]

THE five divergent parabolas, species 67 to 71 , of Newton's Enumeratio Linearum tertii Ordinis (1704), are included unde: the general equation $y^{2}=a x^{3}+3 b x^{2}+3 c x+d$; there are two general forms, or forms without singularities, viz. the parabola cum ovali, sp. 67 , and the parabola pura, sp. 71 ; two forms having a double point, viz. the nodata, sp. 68, and the punctata, sp. 69, according as the double point is one with real branches, or is a conjugate or isolated point; and finally the cuspidata or semicubical parabola, sp. 70, which has a cusp. In the nomenclature of my short note "On Curves of the Third Order," British Assoc. Report for the Year 1861, Notices \&c. p. 2, the five parabolas are the complex, the simplex, the crunodal, the acnodal, and the cuspidal; the distinction there made of the simplex kind of cones of the third order into three subspecies, applies to the simplex parabola, and for this particular case was, as I have since ascertained, noticed in Murdoch's Newtoni Genesis Curvarum per Umbras, 8vo. Lond. 1746, pp. 1-126. It may be remarked that in this very interesting and valuable work the number of species is given as 78 , viz. the author includes the four species added by Stirling, and the other two usually considered to have been added by Cramer (one of them the author himself attributes to Cramer), and that the demonstration of Newton's theorem is effected in the most complete way by showing in what manner the five cones are each of them to be cut so as to obtain the 78 species of cubic curves.

The analytical investigation of the points of inflexion of the above-mentioned divergent parabolas, that is, the curves defined by the equation

$$
y^{2}=a x^{3}+3 b x^{2}+3 c x+d,
$$


is not without interest. The result which should be obtained is, by the general theory of cubic curves, known to be as follows: there is always an inflexion at infinity, in the point where the line infinity is met by the line $x=0$ (or, what is the same thing, by any ordinate of the curve); but disregarding altogether this inflexion at infinity, then in the general case where the curve is without singularity, the remaining eight inflexions (two of them real, six imaginary) lie in pairs on four ordinates of the curve: if however the curve has an acnode, the six imaginary inflexions coincide with the acnode, viz. the three ordinates corresponding to these pass through the acnode, but there are still two real inflexions; if the curve has a crunode, four of the imaginary inflexions and the two real inflexions coincide with the crunode, viz. the three ordinates corresponding to these pass through the crunode, and there is not any real inflexion, although there are still two imaginary inflexions; finally, if the curve has a cusp, then the eight inflexions coincide with the cusp, viz. the four ordinates corresponding to these pass through the cusp, and there is no inflexion real or imaginary.

Proceeding now to the analytical investigation, if in order to form the Hessian we introduce the new coordinate $z=1$, the equation of the curve becomes

$$
U=-y^{2} z+a x^{3}+3 b x z^{2}+3 c x z^{2}+d z^{3},
$$

and thence forming the second differential coefficients, and ultimately replacing $z$ by its value, $=1$, we have

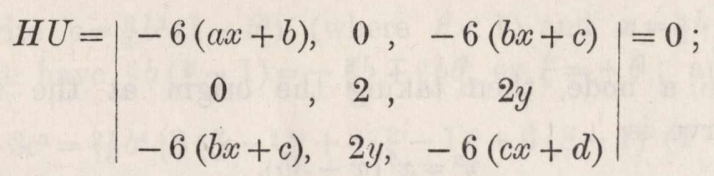

whence, developing and dividing by 24 , we find

$$
3\left\{(a x+b)(c x+d)-(b x+c)^{2}\right\}+(a x+b) y^{2}=0,
$$

or, what is the same thing,

$$
3\left\{\left(a c+b^{2}\right) x^{2}+(a d-b c) x+\left(b d-c^{2}\right)\right\}+(a x+b) y^{2}=0,
$$

as the equation of the Hessian curve, meeting the given cubic curve

$$
a x^{3}+3 b x^{2}+3 c x+d-y^{2}=0,
$$

in its points of inflexion. Multiplying the last-mentioned equation by $b$, and adding it to the equation of the Hessian, we obtain

$$
a b x^{3}+3 a c x^{2}+3 a d x+4 b d-3 c^{2}+a x y^{2}=0,
$$

or, what is the same thing,

$$
x y^{2}=-b x^{3}-3 c x^{2}-3 d x+\frac{3 c^{2}-4 b d}{a},
$$


as the equation of a zurve meeting the given curve

$$
y^{2}=a x^{3}+3 b x^{2}+3 c x+d,
$$

in its points of inflexion; and if for greater simplicity we assume $a=1, d=0$ (the latter equation means obviously that the origin is taken at one of the three intersections of the curve with the axis of $x$, say the real one, if the intersections are one real, two imaginary), then the equation of the curve is

$$
y^{2}=x\left(x^{2}+3 b x+3 c\right),
$$

and the inflexions are given as the intersections of the curve with the curve

$$
x y^{2}=-b x^{3}-3 c x^{2}+3 c^{2} .
$$

There is, it is clear, an inflexion at the point at infinity on the line $x=0$; and eliminating $y^{2}$ we find

$$
x^{4}+3 b x^{3}+3 c x^{2}=-b x^{3}-3 c x^{2}+3 c^{2},
$$

or, what is the same thing,

$$
x^{4}+4 b x^{3}+6 c x^{2}-3 c^{2}=0,
$$

a quartic equation giving the four ordinates through the remaining eight inflexions.

If the curve has a cuspidal point, then the origin will be at the cusp, and we have $b=0, c=0$, and the quartic equation becomes $x^{4}=0$; that is, the four ordinates pass through the cusp.

If the curve have a node, then ta'ing the origin at the node we have $c=0$; the equation of the curve is

$$
y^{2}=x^{2}(x+3 b),
$$

and the curve has a crunode or an acnode according as $b$ is positive or negative; the quartic equation becomes

$$
x^{3}(x+4 b)=0,
$$

and the factor $x^{3}=0$ gives three ordinates through the node; the remaining factor $x+4 b=0$ gives the ordinate through the two inflexions; and substituting this value of $x$ in the equation of the cubic, we find

$$
y^{2}=-16 b^{3}
$$

and the resulting values of $y$ (consequently also the inflexions) are imaginary if $b$ be positive, that is, for the crunodal form; but real if $b$ be negative, that is, for the acnodal form. It is to be observed that the indefinite ordinate $x+4 b=0$ or $x=-4 b$ is real in each of the two cases: in the crunodal case, the ordinate lies outside the curve, that is beyond the loop; in the acnodal case inside the curve, that is on the opposite side to the acnode in regard to the vertex; and using $3 b$ to denote the distance (taken positively) of the vertex from the node, (that is, in the crunodal case changing the sign of $b$ ), the distance (taken positively) of the ordinate from the vertex is $=4 b-3 b,=b,=\frac{1}{3} \cdot 3 b$, that is, it is one-third of the distance of the vertex from the node. 
Consider next the case of a curve without singularities; and first the complex case, the condition for which is that the equation $x^{2}+3 b x+3 c=0$ may have its roots real, or $c<\frac{3}{4} b^{2}$. The values of $x$ which give $y=0$ are

$$
x=0, \quad x=-\frac{3}{2} b \pm \sqrt{ } 3\left(\frac{3}{4} b^{2}-\bar{c}\right) ;
$$

and we may without loss of generality assume that $b$ and $c$ are each of them positive; the value $x=0$ will then belong to the vertex of the parabolic portion, and the two negative values $x=-\frac{3}{2} b \pm \sqrt{3\left(\frac{3}{4} b^{2}-c\right)}$ will belong to the vertices of the oval. The limiting values $c=0$ and $c=\frac{3}{4} b^{2}$ give the acnodal and the crunodal curves respectively, which have been already considered.

In the case in question $\left(b=+, c=+, c<\frac{3}{4} b^{2}\right)$, the equation $x^{4}+4 b x^{3}+9 c x-3 c^{2}=0$ has only two real roots, one of them positive and the other negative; and the positive root substituted in the equation $y^{2}=x\left(x^{2}+3 b x+3 c\right)$ gives $y^{2}=+$, and we have thus the two real inflexions: in order to verify that the negative root gives imaginary inflexions, it must be shown that this negative root does not lie between the two values $x=-\frac{3}{2} b \pm \sqrt{3\left(\frac{3}{4} b^{2}-c\right)}$, or, what is the same thing, that these values substituted for $x$ in the function

$$
x^{4}+4 b x^{3}+3 c x^{2}-3 c^{2},
$$

give results of the same sign.

To verify this write $c=\frac{3}{4} b^{2}\left(1-\theta^{2}\right)$ (where $\theta<1$ ) and $x=\frac{3}{2} b(\xi-1)$; then for. the limiting values of $x$, we have $\frac{3}{2} b(\xi-1)=-\frac{3}{2} b \mp \frac{3}{2} b \theta$, or $\xi= \pm \theta$; and moreover

$$
x^{4}+4 b x^{3}+6 c x^{2}-3 c^{2}=\frac{27}{16} b^{4}\left\{3(\xi-1)^{4}+8(\xi-1)^{3}+6(\xi-1)^{2}\left(1-\theta^{2}\right)-\left(1-\theta^{2}\right)^{2}\right\},
$$

where the term in brackets is

$$
=3 \xi^{3}-4 \xi-6 \theta^{2} \xi^{2}+12 \theta^{2} \xi-4 \theta^{2}-\theta^{4},
$$

and writing $\xi= \pm \theta$, this becomes

$$
-4 \theta^{4}-4 \theta^{2} \pm 8 \theta^{3}, \quad=-4 \theta^{2}(\theta \mp 1)^{2},
$$

so that the two values are each negative, and the theorem is thus proved. It may be added that the curve

$$
y=x^{4}+4 b x^{3}+6 c x^{2}-3 c^{2}
$$

cuts the axis in two real points, one of them situate between the oval and the parabolic portion of the cubic parabola, the other within the parabolic portion.

Lastly, for the simplex case, the condition for which is $c>\frac{3}{4} b^{2}$; the equation $0=x^{4}+4 b x^{3}+6 c x^{2}-3 c^{2}$ has, as before, two real roots, one positive and the other negative; and since the negative root substituted for $x$ in the equation $y^{2}=x^{3}+3 b x^{2}+3 c x$ gives a negative value of $y^{2}$, it is only the positive root which gives an ordinate through two real inflexions. The curve $y=x^{4}+4 b x^{3}+6 c x^{2}-3 c^{2}$ meets the axis in two real points, one of them without, the other within the cubic parabola. 
I remark that the equation $x^{2}+2 b x+c=0$ gives the level points (i.e. the points where the tangent is parallel to the axis) of the cubic parabola. In the complex case, where $c<\frac{3}{4} b^{2}$, then $a$ fortiori $c<b^{2}$ or the values of $x$ are both real, one of these values gives $y^{2}$ positive, and we have thus the maximum ordinate of the oval; the other value of $x$ gives $y^{2}$ negative. In the simplex case, where $c>\frac{3}{4} b^{2}$, we may have $1^{\circ} . c<b^{2}$, and the two values of $x$ give each of them $y^{2}$ positive; the least value of $x$ corresponds to a maximum ordinate, the greatest to a minimum ordinate of the cubic parabola, and between these we have the ordinate through the two real inflexions, the tangents at the inflexions meeting on the axis within the parabola. $2^{\circ}$. We may have $c=b^{2}$, the two values of $x$ here coincide, giving the ordinate through the two real inflexions, the tangents at the inflexions being horizontal. And $3^{\circ}$. We may have $c>b^{2}$, the two values of $x$ are then imaginary and we have no real level point. These are, in fact, Murdoch's three forms, which he distinguishes as the ampullata, media, and campaniformis.

2, Stone Buildings, W.C., June 2, 1863. 